Thelma Larocca Skare ${ }^{1}$

Marcelo Luiz Gehlen²

DandDara Morena Gonçalves Silvere ${ }^{3}$

Mariana Mika de Sousa Uema ${ }^{3}$

Artigo Original

Palavras-chave

Síndromes do olho seco

Ceratoconjuntivite

Aparelho lacrimal/fisiopatologia

Complicações na gravidez

Keywords

Dry eye syndromes

Keratoconjunctivitis

Lacrimal apparatus/physiopathology

Pregnancy complications

\title{
Gravidez e disfunção lacrimal
}

\author{
Lacrimal dysfunction and pregnancy
}

\section{Resumo}

OBJETIVO: Verificar a prevalência de disfunção lacrimal em grávidas, comparando-a com a de mulheres não grávidas. Correlacionar achados de diminuição do filme lacrimal com antecedentes obstétricos. MÉTODOS: Foram entrevistadas 150 mulheres grávidas e 150 não grávidas para avaliação da presença de sintomas de secura ocular e antecedentes obstétricos. Os dois grupos foram submetidos ao teste de Schirmer l e a um questionário para sintomas de olho seco. Pacientes com colagenoses, uso de medicamentos associados à secura de mucosas, hepatite $\mathrm{C}$ e infecção por vírus da imunodeficiência humana, inflamação intraocular prévia ou cirurgia ocular foram excluídas. Os dados obtidos foram analisados por testes de $\chi^{2}$ e Fisher quanto às variáveis nominais, e pelo $t$ de Student e Mann-Whitney quando numéricos. A significância adotada foi de 5\%. RESULTADOS: Os dois grupos não diferiram quanto aos sintomas relacionados à secura ocular. O valor absoluto do teste de Schirmer foi igual nos dois grupos, tanto para olho direito $(p=0,3)$ como esquerdo $(p=0,3)$. Todavia, as mulheres grávidas tiveram maior prevalência de disfunção lacrimal em pelo menos um olho ( $p=0,004)$. A ocorrência de disfunção lacrimal nos dois grupos (pacientes e controles) estava associada a maior número de gestações a termo por paciente $(p=0,04)$, mas não com número de abortos $(p=0,9)$, nem com o tempo da gravidez $(p=0,5)$. CONCLUSÕES: Mulheres grávidas têm mais disfunção lacrimal do que não grávidas. Nos dois grupos a prevalência de disfunção lacrimal é mais alta em mulheres com maior paridade.

\section{Abstract}

PURPOSE: To assess the prevalence of lacrimal dysfunction during pregnancy comparing it to non-pregnant women and to correlate these findings with obstetric history. METHODS: We interviewed 150 pregnant and 150 non-pregnant women for symptoms of dry eyes and obstetric history. Both groups underwent Schirmer I testing and responded to a questionnaire on dry eye symptoms. Patients with collagen diseases, medications associated with dryness of mucous membranes, hepatitis C infection and AIDS, previous intraocular inflammation and eye surgery were excluded. Data were analyzed by the $\chi^{2}$ and Fisher tests when the data were nominal and by the Student's Ftest and Mann-Whitney test when numerical. The level of significance was set at $5 \%$. RESULTS: The two groups did not differ in symptoms of lacrimal dysfunction. The results of Schirmer's test were equal in both groups for the right eye $(p=0.3)$ and left eye $(p=0.3)$. However, pregnant women had a higher prevalence of at least one dry eye $(p=0.004)$. The occurrence of dry eye in both groups (patients and controls) was associated with a greater number of full-term pregnancies/patient $(p=0.04)$ but not with pregnancy time $(p=0.5)$ or number of abortions $(p=0.9)$. CONCLUSIONS: Pregnant women suffer more from lacrimal dysfunction than non pregnant women; in both groups the prevalence of tear dysfunction is more elevated in women with higher parity.

Correspondêncio

Thelma L Skare Centro Médico do Hospital Universitário Evangélico de Curitiba - HUEC Rua Sete de Setembro 4713 - Bate (EPP: 80240-000 Curitiba (PR), Brasil

Recebido

$02 / 01 / 2012$

Aceito com modificacões
Trabalho realizado no Serviço de Reumato-Oftalmologia do Hospital Universitário Evangélico de Curitiba - HUEC - Curitiba (PR), Brasil.

' Serviço de Residência em Reumatologia do Hospital Universitário Evangélico de Curitiba - HUEC - Curitiba (PR), Brasil.

2 Serviço de Residência em Oftalmologia do Hospital Universitário Evangélico de Curitiba - HUEC - Curitiba (PR), Brasil.

${ }^{3}$ Acadêmico de Medicina da Faculdade Evangélica do Paraná - FEPAR - Curitiba (PR), Brasil.

Fonte de financiamento: nenhuma.

Conflito de interesses: não há 


\section{Introdução}

A síndrome do olho seco ou ceratoconjuntivite seca (CCS) danifica a superfície ocular e pode ser causa de sintomas de irritação ocular, aumento no risco de ulcerações e de infecção na córnea, bem como de prejuízo da acuidade visual, causando diminuição na qualidade de vida de seu portador $^{1-3}$. Qualquer alteração na composição lacrimal se deve à disfunção da unidade funcional lacrimal, que é constituída pelas glândulas lacrimais, superfície ocular, pálpebras e nervos sensoriais e motores, em conjunto com o funcionamento adequado do filme lacrimal. Essa unidade responde a influências ambientais, imunológicas e endocrinológicas ${ }^{3}$.

Distúrbios quantitativos e qualitativos do filme lacrimal podem ser encontrados em pacientes com várias formas de distúrbios hormonais, como por exemplo a menopausa e durante o uso de contraceptivos e de reposição hormonal $^{3-5}$. Todavia, os estudos nessa área não trazem resultados claros, uma vez que as respostas hormonais podem ser específicas para o gênero, além de induzir efeitos opostos ou até mesmo antagonistas de acordo com o tecido onde atuam, como: tecidos de Sistema Nervoso Central ligados ao comando da produção lacrimal, glândulas sebáceas como as de Meibomio, sistema imune e processo inflamatório, entre outros ${ }^{6}$.

Achados compatíveis com o olho seco são mais comuns na mulher ${ }^{6}$. Sintomas subjetivos, produção lacrimal, estabilidade da lágrima e inflamação da superfície ocular variam de maneira significante durante o ciclo menstrual e o prejuízo de muitas dessas funções parece atingir um pico durante a fase folicular do ciclo, tanto em mulheres normais como naquelas já com disfunção lacrimal, sendo mais acentuadas nessas últimas ${ }^{7}$.

Levando-se em conta as importantes modificações no organismo materno e as alterações nos níveis de vários hormônios durante a gravidez, conjecturou-se que, durante esta fase, a prevalência de disfunção lacrimal poderia aumentar. Este estudo foi planejado para investigar essa possibilidade.

\section{Métodos}

Este é um estudo transversal, observacional, tipo caso-controle realizado sob aprovação do Comitê de Ética em Pesquisa do Hospital Universitário Evangélico de Curitiba (HUEC), PR. Todas as participantes assinaram Termo de Consentimento Livre e Esclarecido.

Cento e cinquenta mulheres grávidas, sem seleção pela idade gestacional, e 150 pacientes não grávidas (controles) foram examinadas e entrevistadas para obtenção de informações demográficas e de antecendentes obstétricos e queixas referentes a disfunção lacrimal. A amostra de pacientes grávidas foi selecionada de pacientes em consulta para atendimento pré-natal no Serviço de Obstetrícia do HUEC. Os controles foram selecionados entre as pacientes que compareceram para atendimento no Serviço de Ginecologia do mesmo hospital, sendo incluídas (tanto casos como controles) por ordem de chegada e pela disponibilidade em participar do estudo.

Para participar do estudo as mulheres deveriam ter mais de 18 anos e capacidade intelectual para entender o termo de consentimento livre e esclarecido. Foram excluídas: as pacientes em uso de antidepressivos, diuréticos e medicamentos hipotensores, anticolinérgicos ou outro medicamento sabidamente relacionado à redução da produção de lágrima; com história de queimaduras e doenças de pele em região periorbitária; pacientes previamente submetidas a cirurgias oculares ou com história de uveítes, esclerites ou outro processo inflamatório intraocular; pacientes com colagenoses (artrite reumatoide, lúpus eritematoso sistêmico, síndrome de Sjögren, dermatomiosite e esclerodermia); e pacientes com historia de HIV ou hepatite C. Pacientes e controles foram pareadas para idade.

As perguntas realizadas para avaliação subjetiva de disfunção lacrimal foram as constantes no grupo de avaliação para os Critérios Classificatórios Americanos Europeus para síndrome de Sjögren ${ }^{8}$, a saber: (a) Você tem a sensação de areia nos olhos? (b) Você tem sintomas de olho seco diariamente? (c) Você usa colírio lubrificante mais do que três vezes por dia? A seguir, para aquelas com achados subjetivos positivos pediuse que graduassem os sintomas em uma escala visual analógica de zero até dez (sendo pontuado zero quando sem sintomas e dez quando os sintomas eram máximos), e que assinalassem se os sintomas apareceram durante a gravidez.

Pacientes e controles foram submetidas ao teste de Schirmer $\mathrm{I}^{9}$ sem anestésico. Este teste consta na introdução de um papel de filtro de $5 \mathrm{~mm}$ de largura por $35 \mathrm{~mm}$ de comprimento (Ophthalmos ${ }^{\circledR}$ ) no saco conjuntival por cinco minutos, sendo feita a medida das porções úmidas após cinco minutos. Foram considerados positivos (secreção lacrimal reduzida) os testes nos quais não se conseguiu obter até $10 \mathrm{~mm}$ de umidade na fita.

Os dados obtidos foram submetidos a estudo com o emprego de tabelas de frequência e de contingência, sendo usados os testes de $\chi^{2}$ e Fisher para estudo de variáveis nominais (como presença ou não dos sintomas de olho seco) e de Mann-Whitney ou teste $t$ para as numéricas (como idade, valores dos testes de Schirmer, semanas de gestação, números de nascidos vivos, número de gestações e abortamentos). Para medidas de tendência central utilizou-se a média e desvio padrão 
para dados com distribuição gaussiana e a mediana para aqueles com distribuição não gaussiana. A significância adotada foi de $5 \%$. Os cálculos foram feitos com auxilio do software Graph Pad Prism ${ }^{\circledR}$ (San Diego, EUA, 2003), versão 4.0.

\section{Resultados}

A análise da idade da população incluída no estudo mostrou que a média de idade das grávidas era de $28,3 \pm 8,3$ anos e das não grávidas de $27,5 \pm 8,5$ anos, sendo o pareamento considerado adequado $(\mathrm{p}=0,3$; teste $t$ de Student).

A análise dos antecedentes obstétricos mostrou que as pacientes grávidas tinham de uma a oito gestações anteriores (mediana de duas) contra zero a cinco das mulheres do Grupo Controle (mediana de zero com $\mathrm{p}<0,0001$; Mann-Whitney). O número de gestações a termo por mulher era de zero a seis (mediana de um) no grupo das grávidas, e de zero a quatro (mediana de zero) no grupo das não grávidas com $\mathrm{p}<0,0001$ (Mann-Whitney). O número de abortamentos anteriores por mulher era de zero a três (mediana de zero), tanto no grupo das grávidas como no controle $(\mathrm{p}=0,005$; Mann-Whitney).

Ao serem analisados os resultados indicativos de achados subjetivos de disfunção lacrimal foram obtidos os dados mostrados na Tabela 1, na qual pode ser observada a inexistência de diferença nos dois grupos quanto a este aspecto.

Somente 2, de 150 (1,3\%) das pacientes grávidas tiveram a percepção de que o olho se tornou mais seco depois da gravidez.

Ao estudar o valor médio do teste de Schirmer obtido nos dois grupos em olho direito, observouse uma mediana de $28 \mathrm{~mm}$ ( 5 a $35 \mathrm{~mm}$ ) no Grupo Controle, e de $25 \mathrm{~mm}$ ( 4 a $35 \mathrm{~mm}$ ) nas grávidas ( $\mathrm{p}=0,3$; Mann-Whitney). No olho esquerdo, os valores medianos foram de $25 \mathrm{~mm}$ ( 4 a $35 \mathrm{~mm}$ ) nas grávidas e de 26,5 mm ( 4 a $35 \mathrm{~mm}$ ) no Grupo Controle ( $\mathrm{p}=0,3$; teste de Mann-Whitney). Todavia, ao analisar o número de pacientes com pelo menos um olho com disfunção lacrimal indicada pelo teste de Schirmer, observou-se que 26/150 (17,3\%) das grávidas contra 10/150 (6,6\%) das não grávidas apresentavam esse achado positivo $\left(\mathrm{p}=0,004 ; \chi^{2}\right)$.

Por último, analisando-se a presença de pelo menos um olho seco por paciente correlacionando-se com os antecedentes obstétricos obtiveram-se os dados da Tabela 2, na qual é possível notar que o número de filhos nascidos vivos/mulher está associado com ocorrência do olho seco (Figura 1).
Tabela 1. Comparacão entre achados subjetivos de olho seco nas amostras de 150 mulheres grávidas e 150 mulheres não grávidas

\begin{tabular}{|c|c|c|c|c|c|}
\hline & \multicolumn{2}{|c|}{$\begin{array}{c}\text { Grávidas } \\
\mathrm{n}=150\end{array}$} & \multicolumn{2}{|c|}{$\begin{array}{l}\text { Não grávidas } \\
n=150\end{array}$} & \multirow{2}{*}{$\begin{array}{c}\text { Valor } \\
\text { P }\end{array}$} \\
\hline & $n$ & $\%$ & n & $\%$ & \\
\hline Sensação de areia nos olhos & $59 / 150$ & 39,3 & $73 / 150$ & 48,6 & $0,1^{\star \star}$ \\
\hline Sensação de olho seco & $24 / 150$ & 16 & $28 / 150$ & 18,6 & $0,5^{\star \star}$ \\
\hline Usa colírio mais que $3 X$ ao dia & $2 / 150$ & 1,3 & $4 / 150$ & 2,6 & $0,6^{\star \star \star}$ \\
\hline $\begin{array}{l}\text { EVA }\left({ }^{*}\right) \text { de sensação de olho } \\
\text { seco (média) }\end{array}$ & \multicolumn{2}{|c|}{$5,5 \pm 2,3$} & \multicolumn{2}{|c|}{$5,8 \pm 2,6$} & $0,4^{\S}$ \\
\hline
\end{tabular}

"EVA: escala visual analógica para medida dos sintomas (valores de 0 a 10); n:número; ${ }^{* *}$ Teste do $\chi^{2} ;{ }^{* * *}$ Teste de Fisher; ${ }^{\circledR}$ Teste $t$ de Student.

Tabela 2. Comparação entre os antecedentes obstétricos em 150 pacientes grávidas com e sem disfuncão lacrimal

\begin{tabular}{|c|c|c|c|}
\hline & $\begin{array}{c}\text { Com disfunção } \\
\text { lacrimal } \\
n=26\end{array}$ & $\begin{array}{c}\text { Sem disfuncção } \\
\text { lacrimal } \\
n=124\end{array}$ & $\begin{array}{c}\text { Valor } \\
p^{*}\end{array}$ \\
\hline Tempo da gravidez em semanas & $14-38$ & $10-42$ & 0,5 \\
\hline Mediana & 31 & 33 & \\
\hline Número de gestações/mulher & $1-8$ & $1-8$ & 0,1 \\
\hline Mediana & 3 & 2 & \\
\hline Número de gestações a termo/mulher & $0-4$ & 1 & 0,04 \\
\hline Mediana & 2 & & \\
\hline Número de abortamentos/mulher & $0-2$ & $0-3$ & 0,9 \\
\hline Mediana & & 0 & \\
\hline
\end{tabular}

*Teste de Mann-Whitney; n: número

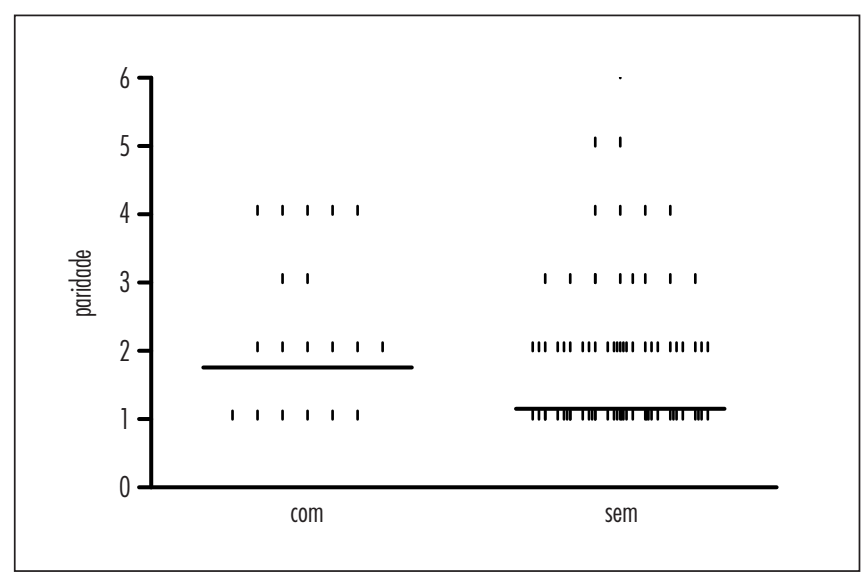

Figura 1. Paridade versus disfunção lacrimal em 150 grávidas com e sem disfunção lacrimal $(p=0,04)$.

\section{Discussão}

A atuação dos hormônios sexuais na unidade funcional lacrimal tem sido objeto de inúmeros estudos, objetivando, inclusive, novas formas de tratamento para essa enfermidade 5 . A testosterona regula o desenvolvimento, a diferenciação e a produção lipídica das glândulas de Meibômio e muitas dessas atuações estão na dependência de ligação com receptores de andrógenos em células acinares e consequente alteração no controle da 
transcrição genética local ${ }^{6,10}$. Os estrógenos, por sua vez, estão associados com redução de tamanho, atividade das glândulas sebáceas e estimulam a liberação lisossômica de enzimas que provocam morte celular e diminuição de produção lipídica. Estrógenos interferem com a conversão de testosterona em dihidrotestosterona e diminuem a captação deste hormônio pelas células ${ }^{6}$.

Todavia, a atuação dos hormônios sexuais sobre o olho não se limita à produção lipídica pelas glândulas de Meibômio. Eles agem sobre processo inflamatório local. Estrógenos estão associados com uma atuação pró-inflamatória resultante de estimulação das enzimas metaloproteinase 3 e catepsina K, além de atuarem sobre fatores de crescimento endotelial, ação essa antagonizada pelos andrógenos ${ }^{6}$.

A análise do desempenho dos hormônios sexuais sobre a função lacrimal da mulher tem resultado em observações controversas 5 . Durante a gravidez, os níveis hormonais maternos são modificados com o objetivo de adaptar a mulher para abrigar o embrião e proporcionar um crescimento adequado ao feto. Os estrogênios estão aumentados, mas também os de andrógenos e progesterona. Entretanto, embora os níveis totais de testosterona estejam aumentados durante a gravidez, deve-se levar em conta que existe, também, aumento concomitante da proteína ligadora desse hormônio (SHBG ou Serum Binding Sexual Hormones), que faz com que a fração livre e biologicamente ativa do hormônio seja bem mais baixa do que o esperado ${ }^{11,12}$. Além disso, tem sido descrito que níveis altos de progesterona contribuem para a sua redução de atuação, uma vez que existe competição entre progesterona e andrógenos pela ligação em receptores celulares ${ }^{11,13}$.

Em animais, comprovou-se maior frequência de olhos secos em coelhas prenhes em comparação com não prenhes. Foi constatada, também, associação desse aumento de frequência com alterações na expressão da aquaporina $4 \mathrm{e}$ da aquaporina 5 , proteínas responsáveis pelo transporte de água em membranas plasmáticas ${ }^{14}$.

No presente estudo não se observou aumento dos sintomas

de secura ocular durante a gravidez. É mister ressaltar que a dissociação entre achados subjetivos e objetivos em olho seco é fato já reconhecido na literatura. A análise do número de pacientes com pelo menos um olho com disfunção lacrimal mostrou que essa entidade está aumentada na gravidez. Sendo esse um achado dissociado das queixas das pacientes, torna-se importante o conhecimento de tal fato pelo médico atendente no sentido de proceder a uma busca ativa do problema e, assim, a instituição precoce de tratamento adequado para se evitar as eventuais complicações. Inesperadamente, verificamos que o número prévio de gestações a termo também pode ser associada com presença da disfunção lacrimal, sugerindo que um efeito residual e somatório pode advir da ocorrência de disfunção lacrimal durante o período gestacional. Esse último achado é inédito. No entanto, e de certa forma correlacionado a este achado, Chubak et al. ${ }^{15}$ demonstraram que a paridade está associada positivamente aos níveis da proteína ligadora de hormônios sexuais (e, consequentemente, com menos andrógenos livres e menor produção lipídica das glândulas de Meibômio).

Este é um estudo exploratório cuja maior falha é a utilização somente do Schirmer para a detecção do olho seco. O uso concomitante do teste de tempo de ruptura do filme lacrimal (TRFL ou BUT = Break Up Time) teria sido interessante, uma vez que tem sido sugerido que esse teste analisa melhor a camada lipídica da lágrima ${ }^{6}$. Uma dissociação entre os resultados obtidos pelo Schirmer e do BUT poderia ser útil na compreensão do papel hormonal sobre as diferentes porções da lágrima. Esse último teste não foi aplicado, pois a presente pesquisa foi realizada na unidade obstétrica do hospital que não dispunha dos instrumentos necessários para essa investigação. No entanto, estudos futuros poderão verificar a correlação entre as duas formas de avaliação.

Concluindo, pode-se afirmar que existe maior prevalência de disfunção lacrimal na gravidez, não evidenciada por achados subjetivos, e que o número de gestações a termo de uma mulher parece associar-se com maior aparecimento dessa disfunção. Maiores estudos são interessantes no sentido de melhor analisar o papel de gestação sobre a unidade funcional lacrimal.

\section{Agradecimentos}

Ao Dr. Augusto F. Beduschi - Chefe do Serviço de Obstetrícia do HUEC, por permitir a realização desta pesquisa nas dependências de seu ambulatório.

\section{Referências}

1. Tabbara KF, Wagoner MD. Diagnosis and management of dry-eye syndrome. Int Ophthalmol Clin. 1996;36(2): $61-75$.

2. Foulks GN. A new appreciation for dry eye disease. Can J Ophthalmol. 2009;44(4):370-2.
3. Fonseca EC, Arruda GV, Rocha EM. Olho seco: etiopatogenia e tratamento. Arq Bras Oftalmol. 2010;73(2):197-203.

4. Erdem U, Ozdegirmenci O, Sobaci E, Sobaci G, Göktolga U, Dagli S. Dry eye in post-menopausal women using hormone replacement therapy. Maturitas. 2007;56(3):257-62. 
5. Versura P, Campos EC. Menopause and dry eye. A possible relationship. Gynecol Endocrinol. 2005;20(5):289-98.

6. Sullivan DA, Jensen RV, Suzuzki T, Richards SM. Do sex steroids exert sex-specific and/or opposite effects on gene expression in lacrimal and meibomian glands? Mol Vis. 2009; 15:1553-72.

7. Versura P, Fresina M, Campos EC. Ocular surface changes over the menstrual cycle in women with and without dry eye. Gynecol Endocrinol. 2007;23(7):385-90.

8. Liquidato BM, Soler RC, Bussoloti Filho I. Avaliação da concordância da sialometria e cintilografia de glândulas salivares em pacientes com boca seca. Rev Bras Otorrinolaringol. 2006;72(1):1 16-9.

9. Daniels TE, Whitcher JP. Association of patterns of labial salivary gland inflammation with kerotoconjuctivitis sicca. Analysis of 618 patients with suspected Sjögren's syndrome. Arthritis Rheum. 1994;37(6):869-77.
10. Duarte MCB, Pinto NT, Moreira H, Moreira ATR, Wasilewski D. Nível de testosterona total em mulheres pós-menopausa com olho seco. Arq Bras Oftalmol. 2007;70(3):465-9.

11. Kaňová N, Bičiková $M$. Hyperandrogenic states in pregnancy. Physiol Res. $2011 ; 60(2): 243-52$

12. Rivarola MA, Forest MG, Migeon CJ. Testosterone, androstenedione and dehydroepiandrosterone in plasma during pregnancy and at delivery: concentration and protein binding. J Clin Endocrinol Metab. 1968;28(1):34-40.

13. Choi JR, Levine $D$, Finberg $H$. Luteoma of pregnancy: sonographic findings in two cases. J Ultrasound Med. 2000;19(12): 877-81

14. Ding $C$, Lu $M$, Huang J. Changes of the ocular surface and aquaporins in the lacrimal glands of rabbits during pregnancy. Mol Vis. $2011 ; 17: 2847-55$. 